\title{
Röntgen: Was muss ich - was darf ich?
}

\author{
Max Giger, Mitglied Zentralvorstand FMH, Winterthur
}

Um gleich zu Beginn ein weitverbreitetes Missverständnis zu klären: Die verschiedenen Vorschriften im Bereich Röntgen beruhen nicht auf Beschlüssen oder Regelungen der FMH, sondern auf der Strahlenschutzgesetzgebung des Bundes. Das Strahlenschutzgesetz (StSG) wurde vom Parlament am 22. März 1991 verabschiedet und zusammen mit der Strahlenschutzverordnung (StSV) am 1. Oktober 1994 in Kraft gesetzt. Am 1. April 1998 folgte die Röntgenverordnung, am 1. Januar 1999 die Strahlenschutz-Ausbildungsverordnung. Für die Umsetzung der Strahlenschutzgesetzgebung auf dem Gebiete der Medizin ist das Bundesamt für Gesundheit verantwortlich.

Da immer wieder Vorwürfe aufkommen, die Interessen der Ärzteschaft in diesem Bereich würden durch die FMH ungenügend wahrgenommen, soll kurz die Geschichte der Strahlenschutzgesetzgebung überflogen werden: Während der parlamentarischen Beratungen wurde von verschiedenen Seiten die Menge der ambulant angefertigten Röntgenaufnahmen und deren Qualität in Frage gestellt. Leider nicht nur zu Unrecht; vorausgehend waren nicht wenige Fälle von offensichtlich unakzeptabler Bildqualität und nicht vertretbarer Indikationsstellungen publik geworden. Und wie es so geht, hat man einmal mehr der konsequenten Aufarbeitung der Einzelfälle die Rasenmäherlösung der Kollektiv-(mass-)regelung aller vorgezogen. In der Konsequenz führte dies in den Arbeitsgruppen, welche für die Erarbeitung der Verordnungen eingesetzt wurden, zu Bestrebungen nicht zuletzt bedingt durch unterschiedliche Interessen der verschiedenen ärztlichen Fachgruppen -, das Röntgen in nichtradiologischen Praxen schlicht und einfach abzuschaffen. In zähen Verhandlungen innerhalb und ausserhalb der FMH gelang es schliesslich, dies zu verhindern, allerdings nur unter zähneknirschendem Akzeptieren der relativ aufwendigen Qualitätssicherungsmassnahmen im apparativen Bereich (Wartung) und der Auflagen im Bereiche der Weiterbildung in Sachverstand und Sachkunde.

Im folgenden soll stichwortartig auf die am häufigsten gestellten Fragen eingegangen werden.

\section{Was ist Sachverstand?}

Sachverstand ist die vertiefte Kenntnis der sicheren Anwendung von ionisierenden Strahlen und deren Auswirkung auf den Menschen sowie Kenntnis der Strahlenschutzgesetzgebung und Fähigkeit zu deren praktischen Anwendung im Alltag.

\section{Welche Ärztinnen und Ärzte müssen über Sachverstand verfügen?}

Alle Ärztinnen und Ärzte, die selbständig eine Röntgenanlage betreiben. Der Sachverständigenausweis ist quasi der Fahrausweis für Röntgenanlagen. In Spitälern und Gruppenpraxen genügt ein Sachverständiger zum Betreiben der Anlagen.

\section{Wie lange gilt der Ausweis für Sachverständige bzw. die Bewilligung zum Betreiben einer Röntgenanlage?} Die nach dem Inkrafttreten des StSG (1. Oktober 1994) erworbenen Sachverständigenausweise sind unbefristet gültig. Inhaber von Bewilligungen zum Betreiben einer Röntgenanlage, die vor Inkrafttreten des StSG erteilt wurden, müssen spätestens bis zum 30. September 2004 einen Sachverständigenkurs (mit Prüfung) oder eine sogenannte zentrale Prüfung über den Sachverstand ablegen, sofern sie weiterhin selbständig röntgen wollen. Der «alte Fahrausweis» verfällt am 30. September 2004.

\section{Was ist Sachkunde?}

Sachkunde ist die Kenntnis der Röntgenanatomie des betreffenden Gebietes und die Kenntnis der Indikationen für entsprechende Röntgenaufnahmen. Das eidgenössische Arztdiplom genügt als Nachweis der Sachkunde für die diagnostische Anwendung von Röntgenanlagen im Bereiche des Niedrigdosisröntgens (Anwendung bzw. Aufnahmen von Thorax und Extremitäten).

\section{Welche Ärztinnen und Ärzte benötigen zusätzliche Sachkunde?}

Einzig für dosisintensive oder interventionelle diagnostische Anwendungen (Aufnahmen des Achsenskelettes und Anwendung von Bildverstärkern und Computertomographen) muss eine 
1 Hänggeli C. Muss ich oder muss ich nicht? Zwang zum Erwerb von Fähigkeits- und Fertigkeitsausweisen? Eine Klarstellung. Schweiz Ärztezeitung 2001; 82(48):2511-3. entsprechende zusätzliche Weiterbildung in Sachkunde absolviert werden. Diese erfolgt entweder im Rahmen der Facharztweiterbildung (u.a. Radiologie, Orthopädie, Urologie, Gastroenterologie) oder im Rahmen der Zusatzweiterbildung für den Fertigkeitsausweis «dosisintensives Röntgen». Zuständig für Programm und Vergabe des Fertigkeitsausweises sind die damit beauftragten Fachgesellschaften (u.a. die Schweizerische Gesellschaft für Kardiologie für Kardiologen, das Kollegium für Hausarztmedizin [KHM] für Grundversorger). Die Programme aller betroffenen Fachbereiche sind auf der Website der FMH (www.fmh.ch/awf) zu finden (vgl. auch die Publikation «Muss ich oder muss ich nicht? Der Zwang zum Erwerb von Fähigkeits- und Fertigkeitsausweisen» [1]).

\section{Wo finden Kurse und Prüfungen statt} und wer beantwortet weitere Fragen?

Im BAG-Bulletin und auf der Website des BAG, Abteilung Strahlenschutz (www.bag.admin.ch), werden die Kurse aufgeführt und weitere Fragen beantwortet.

\title{
Radiologie: que dois-je, que puis-je?
}

\author{
Max Giger, membre du Comité central de la FMH, Winterthour
}

Dissipons d'emblée un malentendu: les diverses prescriptions en matière de radiologie ne se fondent pas sur des décisions ou réglementations de la $\mathrm{FMH}$, mais sur des prescriptions fédérales. La Loi sur la radioprotection (LRaP) a été adoptée par le Parlement le 22 mars 1991 et mise en vigueur, avec l'Ordonnance sur la radioprotection (OraP), le $1^{\text {er }}$ octobre 1994. L'Ordonnance sur les rayons $\mathrm{X}$ a suivi, le $1^{\mathrm{er}}$ avril 1998 et l'Ordonnance sur la formation en radioprotection, le $1^{\text {er }}$ janvier 1999. L'application de la législation sur la radioprotection dans le domaine de la médecine relève de la compétence de l'Office fédéral de la santé publique.

Etant donné les reproches répétés adressés à la FMH, qui ne prendrait pas suffisamment à cœur les intérêts du corps médical dans ce domaine, j'évoquerai ici brièvement l'histoire de la législation sur la radioprotection. Les délibérations parlementaires ont fait apparaître une remise en question de la quantité et de la qualité des clichés radiologiques ambulatoires, pas toujours à tort, hélas. Nombreux étaient en effet les cas rendus publics de mauvaise qualité de l'image et d'indications radiologiques manifestement inacceptables. Dans la foulée, on a préféré, une fois de plus, la solution minimaliste d'une réglementation globale plutôt que d'agir avec conséquence cas par cas. Dès lors, les groupes de travail chargés d'élaborer les ordonnances, largement inspirés - disons-le - par les intérêts divergents des divers groupements de spécialistes, ont mis tous leurs efforts à éliminer purement et simplement la radiologie des cabinets non-radio- logiques. D'âpres négociations à l'intérieur et à l'extérieur de la FMH ont toutefois permis d'enrayer ce processus et d'accepter, avec quelques grincements de dents, il est vrai, des mesures d'assurance-qualité relativement dispendieuses dans le domaine de l'appareillage (maintenance) et de la formation postgraduée pour acquérir la qualité d'expert et les qualifications techniques.

Je réponds ci-après de manière succincte aux questions les plus fréquemment posées.

\section{Qu'entend-on par qualité d'expert?}

C'est le fait de disposer d'une connaissance approfondie de l'utilisation sûre des rayons ionisants et de leurs effets sur l'être humain ainsi que de la législation sur la radioprotection et d'être capable de l'appliquer au quotidien.

\section{Quels sont les médecins devant acquérir cette qualité d'expert?}

Tous les médecins qui exploitent une installation radiologique de manière indépendante. Le certificat d'expert représente quasiment le permis d'exploiter une installation radiologique. Dans les hôpitaux et les cabinets de groupe, un expert suffit pour exploiter les installations.

\section{Quelle est la durée d'un certificat d'expert ou, plus précisément, de l'autorisation d'exploiter une installation radiologique?}

La validité des certificats d'expert acquis après l'entrée en vigueur de la LRaP ( $1^{\text {er }}$ octobre 1994) est illimitée. Les détenteurs d'une autorisation à exploiter une installation radiologique datant 
d'avant l'entrée en vigueur de la LRaP doivent suivre un cours d'expert d'ici au 30 septembre 2004 au plus tard (sanctionné par un examen) ou passer un «examen centralisé d'expert» en radioprotection, dans la mesure où ils souhaitent conserver le droit d'exploiter leur installation radiologique. L'ancien «permis» échoit le 30 septembre 2004.

Qu'est-ce que les qualifications techniques? Les qualifications techniques sont la connaissance de l'anatomie radiologique dans le domaine particulier et la connaissance des indications des clichés radiologiques correspondants. Le diplôme fédéral de médecin suffit pour attester les qualifications techniques concernant l'utilisation diagnostique des installations radiologiques dans le domaine de la radiologie à faibles doses (clichés du thorax et des extrémités).

\section{Quels sont les médecins devant acquérir ces} qualifications techniques supplémentaires?

Une formation postgraduée technique supplémentaire doit être accomplie uniquement pour les applications radiologiques diagnostiques et interventionnelles à fortes doses (clichés du squelette axial, recours aux amplificateurs d'image et à la tomodensitométrie).
Celle-ci s'acquiert soit dans le cadre d'une formation postgraduée de médecin spécialiste (p. ex. en radiologie, orthopédie, urologie, gastroentérologie), soit dans le cadre d'une formation d'appoint donnant droit au certificat d'aptitude technique (CAT) «Qualification pour les examens radiologiques à fortes doses». Sont responsables des programmes et de l'octroi des CAT les sociétés de discipline médicale (p. ex. la Société suisse de cardiologie pour les cardiologues, le Collège suisse de médecine de premier recours [CMPR] pour les médecins de premier recours). Les programmes de toutes les disciplines concernées figurent sur les pages internet de la FMH (www.fmh.ch/index.cfm). Voir aussi la publication: Certificats obligatoires et facultatifs: faut-il ou non acquérir une attestation de formation complémentaire ou un certificat d'aptitude technique?

\section{Où ont lieu les cours et les examens et à qui s'adresser pour des questions supplémentaires?}

Le Bulletin de l'OFSP renseigne sur ces sujets sur son site internet, sous département de la radioprotection (www.bag.admin.ch). 